\title{
Pesquisa operativa para adequação das agendas num serviço de Atenção Primária
}

\author{
Operational research for scheduling adjustment in a primary care service \\ Investigación operativa para adecuación de las agendas en un servicio de atención primaria
}

Ana Maria Sant'Ana ${ }^{a}$

\section{Resumo}

Introdução: Em 2014 a Unidade de Saúde Mãe Curitibana adotou o modelo "carve-out" de organização das agendas, destinando metade das consultas para agendamentos e metade às denominadas consultas de acolhimento, que são reservadas para atendimentos no mesmo dia. Objetivos: Avaliar a proporção de utilização das consultas agendadas e de acolhimento ofertadas. Métodos: Estudo observacional descritivo aplicando formulário de variáveis à totalidade dos pacientes registrados na agenda eletrônica da área denominada "Verde" da Unidade de Saúde Mãe Curitibana, no período de 22 de junho a 24 de julho de 2015. Resultados: Foram 393 mulheres (67\%) e 194 homens (33\%), com $35,43 \%$ das consultas sendo de pessoas acima de 60 anos. Um total de $451(77 \%)$ registros eram de pacientes da área e 136 (23\%) de outras áreas. Houve faltas a 13\% das consultas. A taxa de aproveitamento de consultas (número de comparecimentos/total de consultas ofertadas) foi de $78,77 \%$ (271/344) para as consultas agendadas e $67,89 \%$ (239/352) para os acolhimentos. Dos acolhimentos realizados, $45 \%$ foram destinados a pacientes de outras áreas. Conclusão: A taxa de aproveitamento é maior para as consultas agendadas, demonstrando uma necessidade de ajuste na oferta de consultas. A proporção de pacientes de outras áreas às consultas de acolhimento compromete a continuidade do cuidado.

\section{Abstract}

Introduction: In 2014 "Unidade de Saude Mae Curitibana" (Curitiba Maternal Health Center) adopted the carve-out model of schedule organization, allocating half of the medical appointments as advanced booking and half as same-day appointments. Objectives: To evaluate the application proportion of the scheduled and same-day appointments offered. Methods: Observational descriptive study, applying a variables form to all the patients registered in the electronic agenda of the Green Team of the Curitiba Maternal Health Center from the $22^{\text {nd }}$ of June to the $24^{\text {th }}$ of July 2015 . Results: There were $393(67 \%)$ women and 194 (33\%) men, with $35.43 \%$ of appointments being with people over 60 years old. A total of $451(77 \%)$ were records of the area and $136(23 \%)$ were records of other areas. The application rate of appointments (attendance/total of appointments offered) was 78.77\% (271/344) for scheduled appointments and $67.89 \%$ (239/352) for same-day appointments. Among the same-day appointments, $45 \%$ were delivered for patients from other primary care providers. Conclusion: The application rate is greater for scheduled appointments, demonstrating a necessity to adjust the balance between scheduled and same-day appointments. The proportion of appointments of patients from other providers compromises the continuity of care.

\section{Palavras-chave:}

Acesso aos Serviços de Saúde Agendamento de Consultas

Acolhimento

Assistência Centrada

no Paciente

Continuidade da

Assistência ao Paciente

\section{Keywords:}

Health Services Accessibility Appointments and Schedules Accommodation Patient-Centered Care Patient Care Continuity
Como citar: Sant'Ana AM. Pesquisa operativa para adequação das agendas num serviço de Atenção Primária. Rev Bras Med Fam Comunidade. 2017;12(39):1-9. http://dx.doi.org/10.5712/rbmfc12(39)1459
Fonte de financiamento: declaram não haver. Parecer CEP: 120/2016 (Secretaria Municipal da Saúde de Curitiba), aprovado em 21/12/2016.

Conflito de interesses: declaram não haver. Procedência e revisão por pares: revisado por pares. Recebido em: 31/01/2017. Aprovado em: 26/04/2017. 


\section{Resumen}

Introducción: En el 2014 la "Unidade de Saúde Mãe Curitibana" (Centro de Salud Madre Curitibana) adoptó el modelo carve-out en la organización de los horarios, destinando la mitad de las consultas para los turnos reservados y la otra mitad para las denominadas consultas de acogida, que son reservadas para las atenciones en el mismo día. Objetivos: Evaluar la proporción de utilización de las consultas programadas y de acogida ofrecidas. Métodos: Estudio observacional descriptivo aplicando formulario de variables a la totalidad de los pacientes registrados en la agenda electrónica del área denominada "Verde" de la Unidad de Salud Madre Curitibana, en el período del 22 de junio al 24 de julio de 2015. Resultados: Fueron 393 mujeres (67\%) y 194 hombres (33\%), con el 35,43\% de las consultas siendo de personas con más de los 60 años. Fueron 451 (77\%) registros del área y $136(23 \%)$ de otras áreas. El porcentaje de aprovechamiento de las consultas (número de asistencias/total de consultas ofrecidas) fue del 78,77\% (271/344) para las consultas marcadas y el $67,89 \%$ (239/352) para las acogidas. De los acogidos realizados, el $45 \%$ se destinó a pacientes de otras áreas. Conclusión: El porcentaje del aprovechamiento es mayor para las consultas marcadas, demostrando una necesidad de ajuste en el balance entre consultas marcadas y acogidas. La proporción de pacientes de otras áreas a las consultas de acogida compromete la continuidad del cuidado.
Palabras clave:

Accesibilidad a los

Servicios de Salud

Citas e Horarios

Acogimiento

Atención Dirigida al Paciente

Continuidad de la

Atención al Paciente

\section{Introdução}

A pressão da demanda é um grande desafio para os profissionais de Atenção Primária à Saúde (APS). A cobertura inadequada de serviços contribui para a sobrecarga de trabalho vivenciada pelas equipes, as quais têm buscado novas formas de organização das agendas para garantir o acesso da população.

Nas consultas agendadas, a demanda excedente se acumula no tempo, na forma de filas de espera que podem chegar a meses, no denominado modelo saturado de acesso. ${ }^{1}$ No modelo de acesso avançado, a organização das agendas é considerada mais centrada no paciente, ao preconizar que todos os usuários sejam atendidos no mesmo dia em que procuram a consulta. Exige como premissa uma relação adequada entre a oferta e a procura de serviços..$^{1-3}$

Um modelo intermediário é o chamado de carve out, no qual 50\% das consultas são agendadas e $50 \%$ são reservadas para acesso avançado. A partir de experiência favorável em Florianópolis, ${ }^{4}$ o modelo foi proposto no município de Curitiba ${ }^{5}$ e aplicado a partir de 2014 na Unidade de Saúde da Família (USF) Mãe Curitibana, onde as consultas de acesso avançado são denominadas de "acolhimento".

A Unidade Mãe Curitibana localiza-se no bairro São Francisco e iniciou as atividades como Estratégia de Saúde da Família (ESF) em meados de 2013, contando com 4 equipes que atendem a uma população de 50 mil pessoas em bairros centrais de Curitiba, com indicadores sociais mais favoráveis.

A área de abrangência da Equipe Verde (Área 4) compreende os bairros do Centro Cívico e Bom Retiro, num total de 9.939 pessoas, ${ }^{6,7}$ das quais $3061^{8}(30 \%)$ eram cadastradas no prontuário eletrônico. Os domicílios mais próximos ficam a uma distância de 950m da USF. A Equipe Vermelha é nossa área irmã e somos responsáveis pelos atendimentos no caso de ausência dos profissionais. Sua população é de $4494^{8}$ cadastrados.

À época do levantamento, a Equipe Verde era constituída por: agente comunitária, auxiliar de enfermagem, enfermeira, médica de família e comunidade (MFC), médica residente de primeiro ano em Medicina de Família e Comunidade (R1MFC) e acadêmica de Medicina. A distribuição das consultas da MFC prevê 6 agendamentos de 26 minutos e 8 acolhimentos de 10 minutos por turno, conforme a Tabela 1. A agenda da R1MFC prevê quatro agendamentos pela manhã e três à tarde, e no tempo restante atende acolhimentos da agenda da MFC. 
Tabela 1. Oferta plena de consultas Médicas da Equipe Verde.

\begin{tabular}{lcccccc}
\hline Turno & Tipo & Seg & Ter & Qua & Qui & Sex \\
\hline M & AG & 10 & 10 & 10 & 10 & 10 \\
& AC & 8 & 8 & 8 & 8 & 8 \\
T & AG & 9 & VD & RE & 9 & 9 \\
& AC & 8 & VD & 8 & 8 & 8 \\
\hline
\end{tabular}

M: manhã; T: Tarde; AG: agendamentos; AC: acolhimentos; VD: visita domiciliar; RE: Reunião de Equipe.

Os agendamentos podem ser marcados por telefone ou pessoalmente. Já as consultas para o mesmo dia (consulta de acolhimento) só podem ser solicitadas pessoalmente. Os pacientes são avaliados inicialmente pela enfermeira, que negocia o momento mais oportuno do atendimento médico (consulta agendada ou de acolhimento, de acordo com a condição clínica e a disponibilidade de consultas).

O objetivo deste estudo é realizar um diagnóstico de demanda respondendo a questões sobre a utilização dos tipos de consulta ofertadas (agendadas e de acolhimento) possibilitando a adequação das agendas às necessidades da população.

\section{Materiais e Métodos}

Trata-se de estudo de abordagem quantitativa observacional, com análise das agendas da equipe médica da área denominada "Verde" da Unidade de Saúde Mãe Curitibana durante o período de 22 de junho a 24 de julho de 2015.

O instrumento para coleta de dados foi um formulário de variáveis incluindo data do atendimento, iniciais, sexo, idade, cadastro na Área Verde (Sim/Não), comparecimento à consulta (Sim/Não) e tipo de consulta (agendamento ou acolhimento).

A amostra estudada é do tipo não probabilística, consistindo da totalidade de usuários registrados na agenda médica da Equipe Verde (Área 4) no período. A população residente na área de abrangência que compareceu à consulta no período consiste de 382 pessoas. Para chegar a este número de pessoas da área, foi necessário realizar levantamento de um total de 598 registros, que inclui os atendimentos dos pacientes que não são da área e as agendas dos pacientes que não compareceram à consulta.

Dos 598 registros, foram excluídos 11, que são referentes a um mesmo paciente agendado em 2 ou mais horários seguidos, prática adotada para procedimentos, restando 587 registros para análise. No banco de dados inicial não consta a proporção de consultas segundo período (manhã/tarde), tendo sido elaborada uma tabela à parte.

Para a análise das médias de consultas realizadas pela equipe, foi realizada uma adequação. Como a oferta de consultas ao dia varia em função da realização de atividades fora do consultório como visitas domiciliares e reunião de equipe, avaliamos a média de consultas por turno, eliminando dos cálculos os turnos sem atendimento.

Foram consideradas faltas a consulta de acolhimento os casos em que o paciente foi acolhido pela enfermeira e esta agendou consulta médica na agenda eletrônica, no entanto, o paciente não estava presente ao ser chamado pelo médico. Foram consideradas perdas de consultas de acolhimento os casos em que não houve procura pelas vagas ofertadas. 
Para poder contabilizar as perdas em consultas de acolhimento, recorremos a um expediente que denominamos taxa de aproveitamento de consultas, que consiste no quociente entre o número de comparecimentos e o total de consultas ofertadas. A oferta plena de consultas para o período seria de 385 agendamentos e 360 acolhimentos. Devido a quebras por treinamentos em serviço e atividades próprias da residência médica, o total de ofertas foi de 344 agendamentos e 352 acolhimentos.

Os dados foram digitados no banco de dados Microsoft Excel para Mac 2016. As análises estatísticas descritivas foram realizadas por meio de Tabela dinâmica do programa Microsoft Excel para Mac 2016 e as análises inferenciais foram realizadas no Programa Computacional OpenEpi, um software para estatística epidemiológica de acesso livre online.

A pesquisa foi aprovada pelo Comitê de Ética em Pesquisa da Secretaria Municipal de Saúde da cidade de Curitiba, PR, pelo parecer 120/2016.

\section{Resultados}

Com relação ao sexo, foram registradas 393 mulheres (67\%) e 194 homens (33\%). A população com mais de 60 anos corresponde a $35,43 \%$ dos atendimentos. A distribuição da população por sexo e faixa etária pode ser visualizada no Gráfico 1. A faixa etária com maior número absoluto de consultas é a de 60 a 69 anos. Entre as mulheres de 20 a 39 anos (112), 40 consultas foram relacionadas à gestação e pós-parto $(35,71 \%)$.

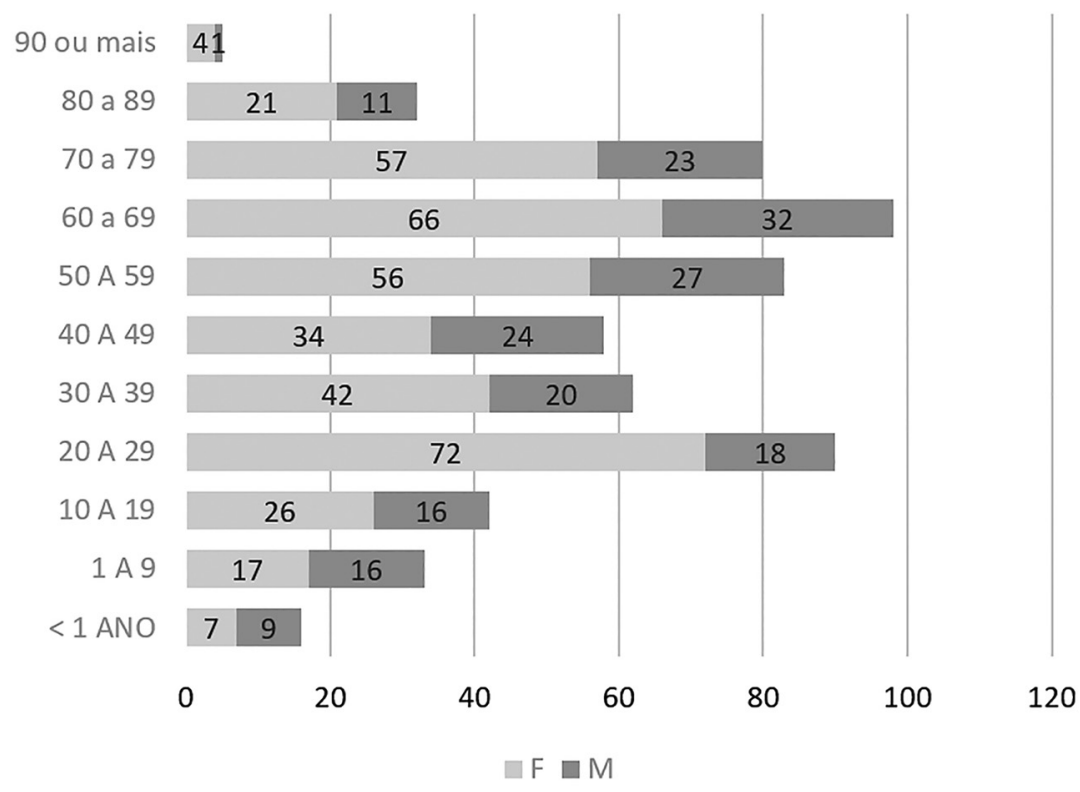

Gráfico 1. Distribuição da população segundo sexo e idade.

As 587 consultas correspondem a 477 pessoas, com média de 1,23 consultas por pessoa. A média de consultas por turno foi de 8,48 ( \pm desvio padrão de 2,84 ) para os agendamentos e 5,52 ( \pm desvio padrão de 2,3) para acolhimentos. Com relação ao dia da semana, houve predominância de consultas às segundas-feiras. 
Houve um total de $77 / 587(13,11 \%)$ faltas às consultas médicas, com média de 3,08 faltas ao dia. Destas faltas, 73/77(94\%) são relativas a consultas agendadas. A procura por consultas de acolhimento apresentou grande variação, indo de 0 a 11 por turno, conforme pode ser observado na Tabela 2. O número de consultas agendadas frequentemente extrapolou o teto de 10 consultas pela manhã e 9 à tarde. A taxa de aproveitamento de consultas foi de 78,77\% (271/344) para as consultas agendadas e 67,89\% (239/352) para os acolhimentos, com risco relativo de 1,35 (Intervalo de Confiança de 95\% de 1,11 a 1,64; $p<0,01$ ).

Tabela 2. Distribuição das consultas segundo dia da semana e turno.

\begin{tabular}{|c|c|c|c|c|c|c|c|c|}
\hline Semana & Turno & Tipo de Consulta & Segunda & Terça & Quarta & Quinta & Sexta & Total \\
\hline \multirow{4}{*}{$\begin{array}{l}22 \text { a } 26 \text { de } \\
\text { junho }\end{array}$} & \multirow{2}{*}{ Manhã } & $A G$ & 12 & 12 & 11 & Treinamento & 11 & 46 \\
\hline & & $\mathrm{AC}$ & 6 & 7 & 6 & Treinamento & 4 & 23 \\
\hline & \multirow{2}{*}{ Tarde } & $A G$ & 11 & \multirow{2}{*}{ VD } & $1^{*}$ & 7 & 11 & 30 \\
\hline & & $\mathrm{AC}$ & 6 & & 4 & 10 & 7 & 27 \\
\hline \multirow{4}{*}{$\begin{array}{l}29 \text { de junho } \\
\text { a } 3 \text { de julho }\end{array}$} & \multirow{2}{*}{ Manhã } & $A G$ & 10 & 11 & 12 & 5 & 12 & 50 \\
\hline & & $\mathrm{AC}$ & 6 & 4 & 4 & 6 & 1 & 21 \\
\hline & \multirow{2}{*}{ Tarde } & $A G$ & 10 & \multirow{2}{*}{ VD } & $1^{*}$ & 3 & 10 & 24 \\
\hline & & $\mathrm{AC}$ & 6 & & 6 & 0 & 3 & 15 \\
\hline \multirow{4}{*}{$\begin{array}{l}6 \text { a } 10 \text { de } \\
\text { julho }\end{array}$} & \multirow{2}{*}{ Manhã } & $A G$ & 11 & 7 & 7 & 5 & 6 & 36 \\
\hline & & $A C$ & 7 & 2 & 3 & 3 & 4 & 19 \\
\hline & \multirow{2}{*}{ Tarde } & AG & 10 & \multirow{2}{*}{ VD } & $0^{*}$ & 7 & 5 & 22 \\
\hline & & $\mathrm{AC}$ & 6 & & 5 & 3 & 9 & 23 \\
\hline \multirow{4}{*}{$\begin{array}{l}13 \text { a } 17 \text { de } \\
\text { julho }\end{array}$} & \multirow{2}{*}{ Manhã } & AG & 10 & 9 & 10 & 10 & 6 & 45 \\
\hline & & $\mathrm{AC}$ & 8 & 8 & 4 & 8 & 7 & 35 \\
\hline & \multirow{2}{*}{ Tarde } & $A G$ & 10 & \multirow{2}{*}{ VD } & $0^{*}$ & 8 & 9 & 27 \\
\hline & & $A C$ & 7 & & 5 & 5 & 3 & 20 \\
\hline \multirow{4}{*}{$\begin{array}{l}20 \text { a } 24 \text { de } \\
\text { julho }\end{array}$} & \multirow{2}{*}{ Manhã } & $A G$ & 11 & 9 & 9 & 10 & 10 & 49 \\
\hline & & $A C$ & 11 & 7 & 11 & 4 & 6 & 39 \\
\hline & \multirow{2}{*}{ Tarde } & $A G$ & 6 & \multirow{2}{*}{ VD } & $0^{*}$ & 9 & 9 & 24 \\
\hline & & $\mathrm{AC}$ & 5 & & 7 & 5 & 6 & 23 \\
\hline Total & & & 169 & 76 & 106 & 108 & 139 & 598 \\
\hline
\end{tabular}

AG: agendamento; AC: acolhimento;VD:Visita Domiciliar. * Reunião de equipe. As células com preenchimento indicam os períodos em que a médica residente não estava presente.

Com relação à área de abrangência, 451 (77\%) registros eram de moradores da área e 136 (23\%) eram de outras áreas, conforme disposto na Tabela 3. Em relação às consultas de acolhimento, 109/239 (45\%) foram dedicadas a pacientes de outras áreas. Foram agendadas 25/136 (18,38\%) consultas de pacientes de outras áreas, com $8 / 25$ faltas (32\%).

Segundo o relatório disponível no prontuário eletrônico "Consultas de Usuários Definitivos Realizadas nas UPAS de $2^{\mathrm{a}}$ a 6ํㅜ feira das 8:00 às 17:00 horas", as consultas realizadas nas Unidades de Pronto Atendimento dos pacientes cadastrados na Unidade de Saúde Mãe Curitibana somaram 418 atendimentos nos meses de junho e julho de 2015. Este relatório não discrimina a microárea do paciente. 
Tabela 3. Oferta de consultas e distribuição da população segundo área, tipo de consulta e faltas.

\begin{tabular}{|c|c|c|c|}
\hline \multicolumn{4}{|c|}{ Oferta de Consultas } \\
\hline Agendadas & & & $344(49,42 \%)$ \\
\hline Acolhimentos & & & $352(50,57 \%)$ \\
\hline \multicolumn{4}{|c|}{ Comparecimentos } \\
\hline & Área & Fora de Área & Total \\
\hline Agendadas & $252(66 \%)$ & $19(15 \%)$ & $271(53 \%)$ \\
\hline Acolhimentos & $130(34 \%)$ & $109(85 \%)$ & $239(47 \%)$ \\
\hline Subtotal (a) & $382(100 \%)$ & $128(100 \%)$ & $510(100 \%)$ \\
\hline \multicolumn{4}{|c|}{ Faltas } \\
\hline Agendadas & $67(97 \%)$ & $6(75 \%)$ & $73(94 \%)$ \\
\hline Acolhimentos & $2(3 \%)$ & $2(25 \%)$ & $4(6 \%)$ \\
\hline Subtotal (b) & $69(100 \%)$ & $8(100 \%)$ & 77 (100\%) \\
\hline Total Geral $(a+b)$ & 451 & 136 & 587 \\
\hline
\end{tabular}

\section{Discussão}

Resumo dos principais achados do estudo

A frequência absoluta de consultas agendadas é maior que a de acolhimentos. Houve faltas em $13 \%$ do total de consultas, sendo que, destas, $94 \%$ ocorreram em consultas agendadas. Houve maior aproveitamento de consultas agendadas que de acolhimentos. Os moradores de outras áreas utilizaram $23 \%$ do total de atendimentos. No entanto, para as consultas de acolhimento a proporção sobe para $45 \%$, comprometendo a continuidade do cuidado.

Fortalezas e limitações do estudo

O estudo apresenta as limitações inerentes aos estudos de demanda e avalia como a população que teve acesso ao serviço se comporta em relação às formas ofertadas de consultas médicas, subsidiando a reorganização das agendas da equipe. O período de seguimento é curto e não caracteriza um estudo rigoroso da demanda, que exigiria idealmente 18 meses. $^{3}$

Comparação com a literatura já existente

O tamanho da população adscrita (3061 pessoas) ultrapassa os limites ideais estabelecidos para o acesso avançado e talvez isto explique o fato da procura por consultas de acolhimento ser de pouca previsibilidade. Em seu artigo clássico em 2000, Mark Murray já alertava para a necessidade de limites apropriados para o tamanho da população, a fim de garantir o equilíbrio entre a oferta e a demanda. ${ }^{1}$

A proporção de faltas é muito variável na literatura. Em um estudo no Rio de Janeiro, as faltas por consultas agendadas chegam a 48,9\%, ${ }^{9}$ valor bem superior ao encontrado em nossa realidade, o que poderia ser explicado por diferenças socioculturais. Em clínicas de atenção primária de Genebra, os pacientes faltam a $22 \%$ das consultas. ${ }^{10}$ No sistema de saúde britânico $6,5 \%$ das consultas são perdidas por faltas. ${ }^{11}$ Nos EUA a prevalência de faltas varia de 5 a $55 \% .{ }^{12,13}$ 
Uma das vantagens do acesso avançado é a redução de faltas, ${ }^{1,14}$ embora no modelo de carve-out isto nem sempre ocorra. ${ }^{15} \mathrm{~A}$ subutilização das consultas de acolhimento foi um resultado imprevisto, porém descrito no modelo de carve-out, caso a demanda para este tipo de consulta fosse superestimada. ${ }^{16}$

A maior utilização de consultas agendadas não encontrou paralelo na bibliografia nacional, mas uma experiência internacional neste sentido merece ser mencionada. O Sistema Nacional de Saúde britânico definiu em 2004 que o tempo máximo de espera para consultar com um médico generalista deveria ser de 48 horas. ${ }^{17}$ Para garantir o cumprimento da meta, os serviços reservaram todos os atendimentos para acesso avançado e deixaram de oferecer consultas agendadas. Os pacientes, insatisfeitos, valorizaram mais poder decidir quando consultar do que ser atendidos prontamente. ${ }^{14,18-20}$

Numa revisão sistemática publicada em 2011, sete entre os nove artigos estudados não encontraram comprometimento da continuidade. ${ }^{9}$ Mas há estudos nos quais, à semelhança do nosso, com o acesso avançado a consulta com o provedor habitual não foi garantida. ${ }^{14,21,22}$

Em nosso serviço, o acesso avançado resultou em maior oferta de consultas, pela menor duração das consultas de acolhimento, com consequente sobrecarga de trabalho. Este acréscimo foi interpretado de forma positiva descrita como aumento da produtividade médica por dois autores, ${ }^{4,14}$ enquanto que um terceiro relatou resultado idêntico como falha na redução da carga de trabalho. ${ }^{22}$ Um estudo dinamarquês encontrou um risco três vezes maior de burnout em médicos que trabalham com acesso avançado. ${ }^{23}$ Outro aponta que recepcionistas e coordenadores avaliam a experiência de forma mais positiva enquanto para os médicos as percepções são ambivalentes. ${ }^{24}$

Um artigo brasileiro alerta sobre a importância de proteger as equipes garantindo que a responsabilidade pelo acesso seja limitada aos pacientes da área de abrangência. ${ }^{25}$ Um artigo britânico de 2006, ao estudar 391 serviços de atenção primária, constatou que a maioria dos profissionais acreditava ter atingido o acesso avançado, mesmo que isso não se constatasse em avaliação mais aprofundada. ${ }^{26}$

\section{Implicações para a pesquisa na área e/ou para a prática dos profissionais}

A seguir listamos os ajustes necessários à organização do serviço segundo os problemas identificados pela pesquisa:

1. Reorganizar as agendas reduzindo a oferta de acolhimentos.

2. Reservar mais consultas de acolhimento para os dias de maior procura.

3. Planejar ações para reduzir o número de faltas às consultas agendadas e garantir mecanismos que facilitem o cancelamento de consultas.

4. Garantir uma proporção adequada de profissionais em relação à população.

5. Assegurar uma distribuição equitativa da população entre as equipes para dividir a sobrecarga de trabalho e garantir a continuidade dos atendimentos.

6. Rever os critérios de vulnerabilidade das populações, que são determinantes para a alocação de recursos, incluindo como critério o atendimento a populações envelhecidas.

7. Garantir que os sistemas informatizados liberem acesso a informações relevantes ao planejamento local das equipes. 


\section{Conclusão}

O termo adotado para designar as consultas médicas do mesmo dia, em nosso meio, foi acolhimento. O termo já havia sido incorporado à terminologia da Saúde no Brasil nos anos 1990, para assegurar que todos os usuários saiam com algum tipo de resposta do serviço de saúde, ainda que não seja a consulta médica, e surgindo também como um contraponto à organização dos serviços direcionada a ações programadas em detrimento da demanda espontânea. ${ }^{27}$

Acesso avançado é um modelo que foi importado de realidades nas quais a cobertura de serviços de saúde é adequada. Em nosso meio, reservar consultas para atendimento no mesmo dia é uma forma de humanizar o atendimento. No entanto, é preciso ter cautela, pois a procura por estas consultas não tem uma distribuição uniforme, com subutilização dos recursos em alguns períodos, ou preenchimento das agendas por pacientes fora de área, em que se compromete a continuidade do cuidado para garantir o acesso.

Para se preservar, as equipes precisam dispor de ferramentas que facilitem o acesso a informações sobre o processo de assistência à saúde, para que possam superar as dificuldades operacionais e promover a adequação necessária. ${ }^{28}$

\section{Referências}

1. Murray M, Tantau C. Same-day appointments: exploding the access paradigm. Fam Pract Manag. 2000;7(8):45-50.

2. Murray M, Berwick DM. Advanced access: reducing waiting and delays in primary care. JAMA. 2003;289(8):1035-40. DOI: http://dx.doi. org/10.1001/jama.289.8.1035

3. Murray M, Davies M, Boushon B. Panel size: how many patients can one doctor manage? Fam Pract Manag. 2007;14(4):44-51.

4. Vidal TB. O acesso avançado e sua relação com o número de atendimentos médicos em atenção primária à saúde [Dissertação]. Porto Alegre: Universidade Federal do Rio Grande do Sul; 2013. 87 p.

5. Prefeitura Municipal de Curitiba/Secretaria Municipal da Saúde de Curitiba/Departamento de Atenção Primária à Saúde/Coordenação do Acesso e Vínculo. Novas possibilidades de organizar o acesso e a agenda na atenção primária à saúde. Curitiba: Prefeitura Municipal de Curitiba; 2014 [acesso 2017 Jun 1]. Disponível em: http://www.saude.curitiba.pr.gov.br/images/cartilha\%20acesso\%20 avan\%C3\%A7ado\%2005_06_14.pdf

6. Instituto de Pesquisa e Planejamento Urbano de Curitiba - IPPUC. Nosso Bairro: Centro Cívico. Lucimara Wons, coordenadora. Curitiba: IPPUC, 2015. [acesso 2016 Set 8] Disponível em: http://ippuc.org.br/nossobairro/anexos/03-Centro\%20C\%C3\%ADvico.pdf

7. Instituto de Pesquisa e Planejamento Urbano de Curitiba - IPPUC. Nosso Bairro: Bom Retiro. Lucimara Wons, coordenadora. Curitiba: IPPUC, 2015. [acesso 2016 Set 8]. Disponível em http://ippuc.org.br/nossobairro/anexos/13-Bom\%20Retiro.pdf

8. Secretaria Municipal de Saúde. Curitiba. Prontuário Eletrônico e-Saúde. Relatório Domicílio Usuário Sintético [acesso em 06/07/15]. Disponível em http://www.saude.curitiba.pr.gov.br/

9. Izecksohn M, Ferreira J. Falta às consultas médicas agendadas: percepções dos usuários acompanhados pela Estratégia de Saúde da Família, Manguinhos, Rio de Janeiro. Revista Brasileira de Medicina de Família e Comunidade. 2014;9(32):235-41. DOI: http://dx.doi. org/10.5712/rbmfc9(32) 960

10. Perron NJ, Dao MD, Kossovsky MP, Miserez V, Chuard C, Calmy, et al. Reduction of missed appointments at an urban primary care clinic: a randomised controlled study. BMC Fam Pract. 2010;11:79. DOI: http://dx.doi.org/10.1186/1471-2296-11-79

11. Martin C, Perfect T, Mantle G. Non-attendance in primary care: the views of patients and practices on its causes, impact and solutions. Fam Pract. 2005;22(6):638-43. DOI: http://dx.doi.org/10.1093/fampra/cmi076 
12. George A, Rubin G. Non-attendance in general practice: a systematic review and its implications for access to primary health care. Fam Pract. 2003;20(2):178-84. DOI: http://dx.doi.org/10.1093/fampra/cmi076

13. Kaplan-Lewis E, Percac-Lima S. No-Show to primary care appointments: why patients do not come. J Prim Care Community Health. 2013;4(4):251-5. DOI: http://dx.doi.org/10.1177/2150131913498513

14. Rose KD, Ross JS, Horwitz LI. Advanced access scheduling outcomes: a systematic review. Arch Intern Med. 2011;171(13):1150-9. DOI: http://dx.doi.org/10.1001/archinternmed.2011.168

15. Bennett KJ, Baxley EG. The effect of a carve-out advanced access scheduling system on no-show rates. Fam Med. 2009;41(1):51-6.

16. Murray M. Answers to your questions about same-day scheduling. Fam Pract Manag. 2005;12(3):59-64.

17. Salisbury C. Does advanced access work for patients and practices? Br J Gen Pract. 2004;54(502):330-1.

18. Salisbury C, Goodall S, Montgomery AA, Pickin DM, Edwards S, Sampson F, et al. Does Advanced Access improve access to primary health care? Questionnaire survey of patients. Br J Gen Pract. 2007;57(541):615-621.

19. Gerard K, Salisbury C, Street D, Pope C, Baxter H. Is fast access to general practice all that should matter? A discrete choice experiment of patients' preferences. J Health Serv Res Policy. 2008;13 Suppl 2:3-10. DOI: http://dx.doi.org/10.1258/jhsrp.2007.007087

20. Kiran T, O’Brien P. Challenge of same-day access in primary care. Can Fam Physician. 2015;61(5):399-400.

21. Phan K, Brown SR. Decreased continuity in a residency clinic: a consequence of open access scheduling. Fam Med. 2009;41(1):46-50.

22. Salisbury C, Montgomery AA, Simons L, Sampson F, Edwards S, Baxter H, et al. Impact of Advanced Access on access, workload, and continuity: controlled before-and-after and simulated-patient study. Br J Gen Pract. 2007;57(541):608-14.

23. Vedsted P, Sokolowski I, Olesen F. Open Access to General Practice Was Associated with Burnout among General Practitioners. Int J Family Med. 2013;2013:383602. DOI: http://dx.doi.org/10.1155/2013/383602

24. Ahluwalia S, Offredy M. A qualitative study of the impact of the implementation of advanced access in primary healthcare on the working lives of general practice staff. BMC Fam Pract. 2005;6:39. DOI: http://dx.doi.org/10.1186/1471-2296-6-39

25. Tesser CD, Poli Neto P, Campos GWS. Acolhimento e (des)medicalização social: um desafio para as equipes de saúde da família. Ciênc Saúde Coletiva. 2010;15(Suppl 3):3615-24. DOI: http://dx.doi.org/10.1590/S1413-81232010000900036

26. Goodall S, Montgomery A, Banks J, Sampson F, Pickin M, Salisbury C. Implementation of Advanced Access in general practice: postal survey of practices. Br J Gen Pract. 2006;56(533):918-23.

27. Brasil. Ministério da Saúde. Secretaria de Atenção à Saúde. Departamento de Atenção Básica. Acolhimento à Demanda Espontânea. Brasília: Ministério da Saúde;2011. [acesso 2017 Jun 1]. Disponível em: http://189.28.128.100/dab/docs/publicacoes/geral/miolo_CAP_28. pdf

28. Takeda S. A Organização de Serviços de Atenção Primária à Saúde. In: Duncan BB, Schmidt MI, Giugliani ERJ, Duncan MS, Giugliani C. Medicina Ambulatorial. Condutas de Atenção Primária Baseadas em Evidências. Porto Alegre: Artmed; 2013. p. 19-32.

${ }^{a}$ Médica de Família e Comunidade do Município de Curitiba. Curitiba, PR, Brasil. anuchas@uol.com.br (Autora correspondente) 